\title{
Seroprevalence of Hepatitis $A$ and Hepatitis $E$ Viruses among Pregnant Women in Northern Iran
}

\author{
Farzin Sadeghi $\left(\mathbb{D},{ }^{1}\right.$ Zahra Golchob, ${ }^{2}$ Maryam Javadian $\left(\mathbb{D},{ }^{3}\right.$ Mohammad Barary $\left(\mathbb{D},{ }^{2}\right.$ \\ Parisa Sabbagh, ${ }^{4}$ Soheil Ebrahimpour ${ }^{(D)},{ }^{4}$ and Masomeh Bayani ${ }^{4}{ }^{4}$ \\ ${ }^{1}$ Cellular and Molecular Biology Research Center, Health Research Institute, Babol University of Medical Sciences, Babol, Iran \\ ${ }^{2}$ Student Research Committee, Babol University of Medical Sciences, Babol, Iran \\ ${ }^{3}$ Department of Gynecology and Obstetrics, School of Medicine, Babol University of Medical Sciences, Babol, Iran \\ ${ }^{4}$ Infectious Diseases and Tropical Medicine Research Center, Health Research Institute, Babol University of Medical Sciences, \\ Babol, Iran
}

Correspondence should be addressed to Masomeh Bayani; dr.bayani1@yahoo.com

Received 12 April 2021; Accepted 28 June 2021; Published 3 July 2021

Academic Editor: Faustino R. Perez-Lopez

Copyright (c) 2021 Farzin Sadeghi et al. This is an open access article distributed under the Creative Commons Attribution License, which permits unrestricted use, distribution, and reproduction in any medium, provided the original work is properly cited.

Background. Hepatitis A (HAV) and hepatitis E viruses (HEV) are endemic in Iran and are known major causes of acute viral hepatitis. Also, during pregnancy, they are associated with severe outcomes. Therefore, it is vital to evaluate the antibody levels against HAV and HEV in pregnant women to avoid severe outcomes incidence. Study design and methods. A total of 247 pregnant women were enrolled in this prospective cross-sectional study. In addition to completing the questionnaire and interviewing all participants, the serum samples were tested for anti-HAV and anti-HEV IgG using the enzyme-linked immunosorbent assay (ELISA). The association between anti-HAV and anti-HEV antibodies status and risk factors was evaluated. Results. The mean age of patients was 28.06 \pm 5.29 years. Anti-HAV antibody was found in 111 patients (44.9\%), while anti-HEV antibody was detected in only two pregnant women (0.8\%). The seroprevalence of HAV was inversely related to the level of education. There was no significant correlation between HAV antibody levels and age, marital status, residence location, and pregnancy trimesters. Conclusion. Considering many complications of these diseases in pregnancy, the detection of enteroviral hepatitis, especially HAV in pregnant women, is necessary, and therefore, proactive measures, such as promoting education, improving people awareness, and vaccination, are recommended.

\section{Introduction}

The principal causes of viral hepatitis are hepatitis A, B, C, and E viruses [1-4], among which the hepatitis A virus (HAV) and the hepatitis E virus (HEV) cause self-limiting acute hepatitis. These viruses spread via the fecal-oral route or by ingestion of contaminated water or food [5]. In many developing countries, these infections' prevalence is closely associated with poor hygiene conditions and low socioeconomic status. In recent years, the incidence of HAV and HEV has decreased markedly due to improved health conditions. The risk of infection in youth was more significant than in adults [6]. Also, there was a high risk of vertical transmission of these viruses from the mother to the fetus with subsequent maternal and fetal complications, such as abortion, neonatal death, and premature labor $[7,8]$. Thus, the diagnosis of these infections in pregnant women is vital to avoid any adverse outcomes. Laboratory results indicating an increase in anti-HAV or anti-HEV IgG levels in serum indicate past exposure or acute infection by these infections.

The levels of IgG antibodies in some asymptomatic populations related to HAV and HEV infections vary significantly. These levels are particularly variable among asymptomatic pregnant women. Besides, there are numerous reports that Iran is located in an endemic area of HAV infection, and several studies in Iran have shown that the HAV seroconversion 
rates are high among young Iranians [9]. Also, some reports indicated that the overall seroprevalence of $\mathrm{HEV}$ in Iran ranged from $2 \%$ to more than $40 \%$ [10].

With all this said, this study is aimed to measure the seroprevalence of anti-HAV and anti-HEV antibodies and potential risk factors among pregnant women referred to Ayatollah Rouhani Hospital in Babol, Northern Iran.

\section{Materials and Methods}

2.1. Patients. This prospective cross-sectional study was comprised of 247 pregnant women aged 17-42 years. Pregnant women with no history of immunosuppressive diseases, such as human immunodeficiency viruses (HIV), cancers, chronic liver diseases, and use of corticosteroids, were referred to Ayatollah Rouhani Hospital in Babol, Northern Iran, between March and December, 2020, for their standard check-up services were enrolled. A standardized questionnaire was used to collect information on social, demographic data (age, education, place of residence), parity, and pregnancy trimesters. Before participating, the study's purpose was clearly explained for all pregnant women, and written informed consent was obtained from all individuals.

2.2. Laboratory Analysis. Blood samples (5 mL) were collected from each participant, centrifuged, and separated serum was stored at $-20^{\circ} \mathrm{C}$ until further analysis. It is noteworthy that all serum samples were duplicated for anti$\mathrm{HAV}$ and anti-HEV total (IgG) antibodies analysis using an enzyme-linked immunosorbent assay (ELISA) commercial Kit (Dia.Pro Diagnostic Bioprobes Srl, Milan, Italy). For the anti-HEV IgG ELISA kit, the limit of detection and sensitivity was $<0.1 \mathrm{IU} / \mathrm{mL}$ and $0.25 \mathrm{IU} / \mathrm{mL}$, respectively. Regarding the anti-HAV IgG ELISA kit, these characteristics were $<0.01$ $\mathrm{IU} / \mathrm{mL}$ and $0.15 \mathrm{IU} / \mathrm{mL}$, respectively.

2.3. Statistical Analysis. Statistical analysis was performed using the SPSS software v. 16.0 (IBM, Chicago, IL, USA). A Chi-square test was used to compare anti-HEV/HAV seroprevalence for the mentioned stratification factors. A $p$ value $<0.05$ was considered to be statistically significant.

\section{Results}

Table 1 presents the demographic data of the study participants. Of the 247 pregnant women, 111 (44.9\%) were positive for total anti-HAV IgG, and $2(0.8 \%)$ were positive for antiHEV IgG antibody. The overall mean age of pregnant women was $28.06 \pm 5.29$ years, with a range of $17-42$ years. Besides, the mean ages of positive anti-HAV and anti-HEV antibodies were $27.9 \pm 5.18$ and $28.25 \pm 5.43$ years, respectively. Also, there was a strong inverse statistical association between education status and seroprevalence of $\operatorname{HAV}(p=0.003)$. Our data showed that HAV incidence was higher in rural regions and among women in their second trimester followed by third and first trimesters, respectively. Also, the highest rate of HAV infection (49.8\%) was seen in pregnant women with first pregnancy experience compared with pregnant women who had one or more children. Moreover, this infection was observed more in patients $\leq 30$ years of age (Table 2 ). Our
TABLE 1: Demographic characteristics of study patients.

\begin{tabular}{lc}
\hline Demographic characteristics & $\begin{array}{c}\text { Frequency } \\
n(\%)\end{array}$ \\
\hline Age & $148(60)$ \\
$\leq 30$ & $99(40)$ \\
$>30$ & \\
Educational status & $169(68.4)$ \\
No formal education & $78(31.6)$ \\
College and above & \\
Place of residence & $149(60.3)$ \\
Rural & $98(39.7)$ \\
Urban & \\
Parity & $123(49.8)$ \\
First pregnancy & $107(43.3)$ \\
Second & $17(6.9)$ \\
Third or more & \\
Pregnancy trimesters & $69(27.9)$ \\
First & $98(39.7)$ \\
Second & $80(32.4)$ \\
Third &
\end{tabular}

findings also indicate that the $\mathrm{HEV}$-infected patients were both aged $\leq 30$ years, resided in rural areas, had college or above education, were in their third trimester, and had one or more children. It is also noteworthy that a simultaneous infection with HAV and HEV was not observed in any study participants.

\section{Discussion}

HAV and HEV are the leading causes of acute self-limiting viral hepatitis worldwide [11]. The severe form of infection can result in a significant mortality rate in pregnant women [11]. Our study showed that the overall seroprevalence of $\mathrm{HEV}$ infection in pregnant women's study population was $0.8 \%$. Our finding, similar to the rate of HEV in the developed countries, was lower than the seroprevalence of HEV infection among pregnant women in other developing countries, such as India (33.6\%), Sudan (41\%), and Egypt (84.3\%) [11, 12]. Moreover, one study in Spain reported that the rate of HEVpositive IgG was $3.6 \%$ [13]. These differences could be explained by the difference in socioeconomic and educational status, hygiene level, and the endemic status of the virus.

Our results also showed that the overall seroprevalence of HAV infection in the study population for pregnant women was $44.9 \%$, almost similar to a previous study by Farajzadegan et al. in which HAV seroprevalence in Iran was reported to be $51 \%$ [14]. The seroprevalence of HAV varies worldwide. In developed countries, health and vaccination have reduced the incidence of HAV [15]. In Asia, the seroprevalence of HAV varies between high, moderate, and low levels in different regions. It is noteworthy that Iran has been considered an HAV endemic region in recent world reports [16].

We found a significant inverse association between educational status and HAV because HAV-positive women's 


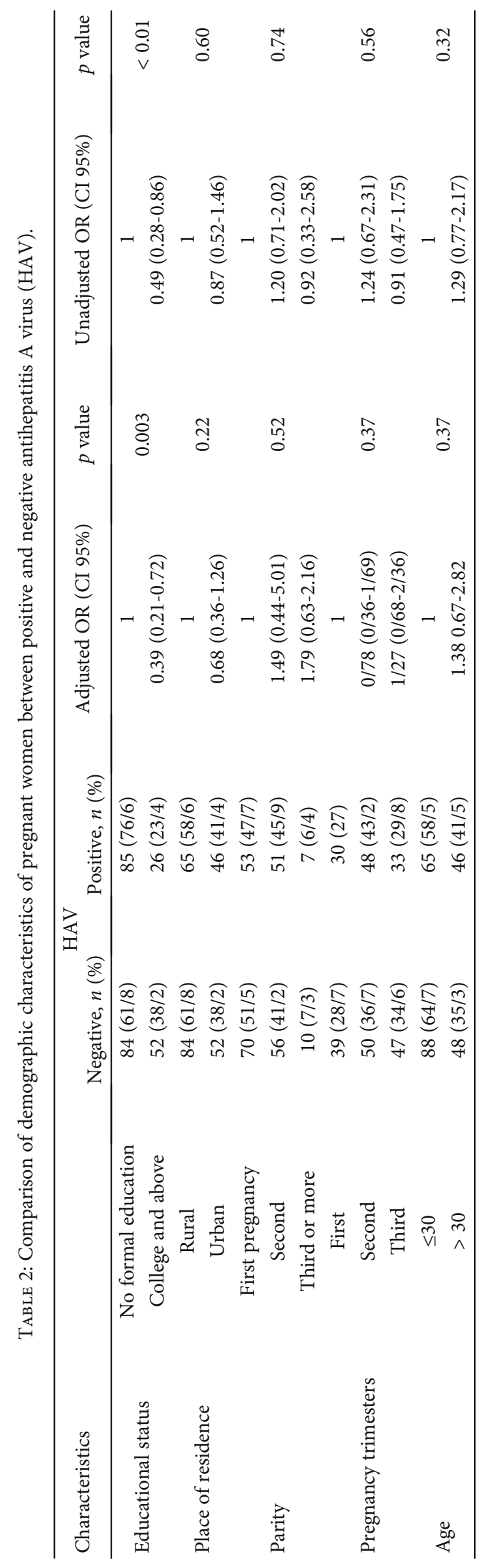


rate increased with lower education levels $(p=0.003)$, which was consistent with a previous study by Ramezani et al. [17]. No significant relationship between age and frequency of HAV was observed due to the proximity of age groups in the present study, although our results showed that the anti-HAV antibodies were more detected in people aged $\leq 30$ years than that of other age groups. These results were in contradiction to the results of a study by Mohebbi et al., where most infected patients were $>30$ years old [18].

While the rate of infection among rural residents was higher than that of urban residents, living in a city or village does not significantly affect the HAV infection rate, which is consistent with a previous study [19]. On the other hand, Caron et al. reported that $\mathrm{HEV}$ was more prevalent in urban areas [20]. The reason for this can be attributed to access to safe drinking water, use of a hygienic toilet system, increasing awareness of the general population, and more sanitation in these areas. It is of interest to mention a strong confounding bias in comparing this stratification factor. Many people now residing in cities lived in the village for many years, especially in their childhood. Therefore, they might have been infected with the HAV in the past. Thus, these patients' past medical history should also be considered, which was possible in this study.

Our results also showed that HAV incidence is higher among women in their second trimester, followed by third and first trimesters, respectively. These findings are consistent with previous studies in which most cases of HAV and $\mathrm{HEV}$ infections were observed in second and third trimesters [21-23]. Moreover, the highest rate of infection was seen in pregnant women with first pregnancy experience compared with pregnant women who had one or more children. Nonetheless, no significant relationship was observed in these regards that might be attributable to the small sample size.

\section{Conclusion}

Due to improved health conditions, the risk of developing hepatitis is reduced in childhood but increased in adulthood. Also, it is crucial to consider the many complications of these diseases during pregnancy. Therefore, the detection of hepatitis $\mathrm{A}$ and $\mathrm{E}$ viruses in pregnant women is important. Thus, ways to promote health problems, such as increasing the awareness of these infections and vaccination in the general population, can prevent adverse complications.

\section{Data Availability}

The data that support the findings of this study are available from the corresponding author upon reasonable request.

\section{Ethical Approval}

This study protocol was approved by the ethics committee of Babol University of Medical Sciences (MUBABOL.HRI.REC.1396.141).

\section{Conflicts of Interest}

All authors declare no conflict of interest.

\section{Acknowledgments}

The authors thank the Department of Infectious diseases of Babol University of Medical Sciences. This study was fully supported by the vice-chancellor for research and technology of Babol University of Medical Sciences.

\section{References}

[1] M. Riaz, M. Idrees, H. Kanwal, and F. Kabir, "An overview of triple infection with hepatitis B, C and D viruses," Virology Journal, vol. 8, no. 1, p. 368, 2011.

[2] M. R. Hasanjani Roushan, S. Farokhtabar, M. Bayani, and S. Siadati, "Epidemiological aspects of hepatitis B and C and human immunodeficiency viruses among hemodialysis patients in Mazandaran Province, Iran," Nephro-urology Monthly, vol. 8, no. 3, article e37878, 2016.

[3] M. Heidari, M. Bayani, A. Bijani, and M. R. Hasanjani Roushan, "Factors influencing therapeutic response to pegylated interferon plus ribavirin in the different genotypes of chronic hepatitis C," Caspian Journal of Internal Medicine, vol. 5, no. 4, pp. 219-222, 2014.

[4] J. Masrour-Roudsari and S. Ebrahimpour, "Causal role of infectious agents in cancer: an overview," Caspian Journal of Internal Medicine, vol. 8, no. 3, pp. 153-158, 2017.

[5] K. A. Traoré, H. Rouamba, Y. Nébié et al., "Seroprevalence of fecal-oral transmitted hepatitis A and E virus antibodies in Burkina Faso," PLoS One, vol. 7, no. 10, article e48125, 2012.

[6] J. Hartl, M. Wehmeyer, and S. Pischke, “Acute hepatitis E: two sides of the same coin," Viruses, vol. 8, no. 11, p. 299, 2016.

[7] A. B. Labrique, L. J. Krain, J. E. Atwell, and K. E. Nelson, "Fetal and neonatal health consequences of vertically transmitted hepatitis E virus infection," The American Journal of Tropical Medicine and Hygiene, vol. 90, no. 2, pp. 365-370, 2014.

[8] Shalimar and S. K. Acharya, "Hepatitis e and acute liver failure in pregnancy," Journal of Clinical and Experimental Hepatology, vol. 3, no. 3, pp. 213-224, 2013.

[9] S. G. Hoseini, R. Kelishadi, B. Ataei et al., "Seroprevalence of hepatitis A in Iranian adolescents: is it time to introduce a vaccine?," Epidemiology and Infection, vol. 144, no. 2, pp. 291296, 2016

[10] A. Esmaeilzadeh, A. Ganji, A. Bahari, and L. Goshayeshi, "Prevalence of hepatitis E in Iran: a systematic review of the literature," Reviews in Clinical Medicine, vol. 4, no. 4, pp. 152159, 2017.

[11] M. Abebe, I. Ali, S. Ayele, J. Overbo, A. Aseffa, and A. Mihret, "Seroprevalence and risk factors of hepatitis E virus infection among pregnant women in Addis Ababa, Ethiopia," PLoS One, vol. 12, no. 6, article e0180078, 2017.

[12] D. Obiri-Yeboah, Y. Asante Awuku, J. Adu et al., "Sero-prevalence and risk factors for hepatitis E virus infection among pregnant women in the Cape Coast Metropolis, Ghana," PLoS One, vol. 13, no. 1, article e0191685, 2018.

[13] M. L. Lindemann, G. Gabilondo, B. Romero, O. M. S. de la Maza, and M. T. Pérez-Gracia, "Low prevalence of hepatitis E infection among pregnant women in Madrid, Spain," Journal of Medical Virology, vol. 82, no. 10, pp. 1666-1668, 2010.

[14] Z. Farajzadegan, S. G. Hoseini, R. Kelishadi et al., "Systematic review and meta-analysis on the age-specific seroprevalence of hepatitis A in Iran," Journal of Research in Medical Sciences : 
the Official Journal of Isfahan University of Medical Sciences, vol. 19, Supplement 1, pp. S56-S63, 2014.

[15] K.-Y. Lin, G.-J. Chen, Y.-L. Lee et al., "Hepatitis A virus infection and hepatitis A vaccination in human immunodeficiency virus-positive patients: a review," World Journal of Gastroenterology, vol. 23, no. 20, pp. 3589-3606, 2017.

[16] M. Izadi, A. A. Esfahani, H. Hassannia, N. Jonaidi Jafari, F. Rahmati Najarkolaei, and M. S. Rezaee-Zavareh, "Seroprevalence of hepatitis A virus among Iranian soldiers," Gastroenterology and Hepatology from Bed to Bench, vol. 9, no. 2, pp. 100-104, 2016.

[17] H. Ramezani, S. H. Bozorgi, M. Nooranipour et al., "Prevalence and risk factors of hepatitis A among blood donors in Qazvin, central Iran," Singapore Medical Journal, vol. 52, no. 2, pp. 107-112, 2011.

[18] S. R. Mohebbi, M. Rostami Nejad, S. M. Tahaei et al., "Seroepidemiology of hepatitis A and E virus infections in Tehran, Iran: a population based study," Transactions of the Royal Society of Tropical Medicine and Hygiene, vol. 106, no. 9, pp. 528531, 2012.

[19] C. Alvarado-Esquivel, L. F. Sánchez-Anguiano, and J. Hernández-Tinoco, "Hepatitis E virus exposure in pregnant women in rural Durango, Mexico," Annals of Hepatology, vol. 13, no. 5, pp. 510-517, 2014.

[20] M. Caron and M. Kazanji, "Hepatitis E virus is highly prevalent among pregnant women in Gabon, central Africa, with different patterns between rural and urban areas," Virology Journal, vol. 5, no. 1, p. 158, 2008.

[21] A. Tejada-Strop, R. A. Tohme, J. Andre-Alboth et al., "Seroprevalence of hepatitis $A$ and hepatitis $E$ viruses among pregnant women in Haiti," The American Journal of Tropical Medicine and Hygiene, vol. 101, no. 1, pp. 230-232, 2019.

[22] E. Elinav, I. Z. Ben-Dov, Y. Shapira et al., "Acute hepatitis A infection in pregnancy is associated with high rates of gestational complications and preterm labor," Gastroenterology, vol. 130, no. 4, pp. 1129-1134, 2006.

[23] N. Urganc, M. Arapoglu, B. Akyldz, and A. Nuhoğlu, "Neonatal cholestasis resulting from vertical transmission of hepatitis A infection," The Pediatric Infectious Disease Journal, vol. 22, no. 4, pp. 381-382, 2003. 